\title{
METASTABLE MAGNETIC STRUCTURES IN THE BLUME-CAPEL MODEL
}

\author{
K. Kulakowski ${ }^{a}$, M. AntoniuK $^{a}$, A. Del Moral ${ }^{b}$ And P. GaWroński ${ }^{a}$ \\ ${ }^{a}$ Faculty of Physics and Nuclear Techniques, University of Mining and Metallurgy \\ Al. Mickiewicza 30, 30-059 Cracow, Poland \\ ${ }^{b}$ Laboratorio de Magnetismo de Solidos \\ Departamento de Fisica de Materia Condensada, Universidad de Zaragoza \\ 50009 Zaragoza, Spain
}

\begin{abstract}
We discuss a first-order phase transition from non-magnetic to magnetic phase at $T=0$. New results are presented on a spontaneously formed magnetic phase and its energy in the $C 15$ atomic structure, in the presence of the antiferromagnetic interaction. A dynamic phase diagram is constructed for the phase transition. The results are discussed in the context of the pressure-driven phase transition, which was observed experimentally in $\mathrm{YMn}_{2}$.
\end{abstract}

PACS numbers: $05.70 . \mathrm{Fh}, 75.10 . \mathrm{Hk}, 75.30 . \mathrm{Kz}$

\section{Introduction}

It is known that rare earth- $\mathrm{Mn}_{2}$ compounds show a magnetic-non-magnetic phase transition at low temperatures [1]. In the non-magnetic phase, above the Néel temperature, magnetic moments of $\mathrm{Mn}$ vanish, which is accompanied by a collapse of volume (see Ref. [2] and references therein). One can observe also an inverse transition, i.e. from the non-magnetic to the magnetic phase, when pressure is reduced at $0 \mathrm{~K}$. Here we intend to discuss the question of the kind of magnetic structure which appears at such an inverse transition. In particular, we are interested in the role of the initial state at the interphase boundary.

The problem is particularly complex when one deals with magnetic structures with many frustrated bonds. That is why we have made several assumptions, which allow us to control the results. Firstly, we assume that the pressure is non-uniform and its gradient is strong enough to form a well-defined boundary between the magnetic and the non-magnetic phase. Secondly, we limit our description to the Blume-Capel model, in which all magnetic moments are aligned parallel to the $z$ axis. Finally, we intend to focus our discussion on $\mathrm{YMn}_{2}$ because yttrium carries no magnetic moment.

Numerical method of this research is twofold. We investigate the connexion between the initial structure of an already magnetic part of a sample and 
the structure which is formed in contact with the initial one during the phase transition. We also check the stability of the new structure with respect to small variations of the initial structure. Preliminary results of the application of the method to $\mathrm{YMn}_{2}$ were already reported in Ref. [3]. Here we give the details of the dynamic phase diagram, which can be derived from the model Hamiltonian for antiferromagnetic manganese sublattice of $\mathrm{YMn}_{2}$. Also we focus on the energy of the magnetic structures, obtained during the transition, and the energy of the damages.

\section{The ground states}

In $C 15$ structure, 16 manganese atoms form four tetrahedra in each elementary cell. The ground state energy of a tetrahedron is equal to $2 J$ and is negative; here $J$ is the exchange integral in the Blume-Capel Hamiltonian. Any flip of any spin enhances the energy of a tetrahedron. Looking for a ground state configuration for the lattice, we have limited the set of states to those which fulfil periodic boundary conditions. We have got 90 such states. There are two spins up and two spins down in each tetrahedron. This rule does not allow energies lower than $16 \mathrm{~J}$ per unit cell. Still the energy could be enhanced by interactions between tetrahedra. However, we find that the energy is exactly $16 \mathrm{~J}$.

We have also checked that to pass from one ground state to another it is necessary to reverse at least four spins in each unit cell. This distance is a measure of the local energy depth of local energy minima. Typically, such a four-spin-flip consists of two mutual flips of antiparallel spins in two neighboring tetrahedra. These flips cause that the ground state degeneracy of the structure is practically unbound. The histogram of the Hamming distances between the obtained ground state configurations is given in Table.

\section{TABLE}

A histogram of the Hamming distance $H$, i.e. number of atoms with different spins, between the ground state configurations. The values of $H$, where the number of pairs is zero, are omitted.

\begin{tabular}{l|c|c|c|c|c|c}
\hline \hline Hamming distance $H$ & 4 & 6 & 8 & 10 & 12 & 16 \\
\hline $\begin{array}{l}\text { Number of pairs of configurations } \\
\text { with mutual distance } H\end{array}$ & 576 & 576 & 1656 & 576 & 576 & 90
\end{tabular}

\section{Dynamic phase diagram}

The dynamic phase diagram allows to assign a cellular automaton, which is a kind of digital equation of motion, to each set of values of the parameters of the Blume-Capel model. The one-site Hamiltonian is $H_{i}=\Delta s_{i}^{2}+J \sum_{j} s_{j}-h s_{i}$, where $s_{i}= \pm 1$ or 0 . If the interphase boundary in the structure $C 15$ is a plane, there are always three neighbours of each atom which are already magnetic. Then, there are seven possibilities of the values of the effective field, from $h=-3$ to +3 . Example giving, the value zero is produced by the configurations $000,0+-$ and by their permutations. The shortest way to present the dynamic phase diagram is 
as follows: in the plane $x=-\Delta / J, y=-h / J$ we restrict our considerations to $J<0, h>0$. For $x<0$ we get seven horizontal lines of the diagram: $y=-3, \ldots, 3$. For the neighbourhood characterized by the effective field equal to $h$, above an $h$-th line the automaton gives the state " + ", and below this line - the state "-". For $x>0$ the diagram consists of 14 lines: $y=h+x$ and $y=h-x$ for each value of $h=-3, \ldots, 3$. For the neighbourhood characterized by the effective field equal to $h$, above the line $y=h+x$ the automaton gives " + ", and below the line $y=h-x$ it gives "-". Between these lines, the output is " 0 ". These rules determine the transition for each values of $J, h$, and $\Delta$.

\section{Metastable structures}

Numerical simulations are performed in two ways. First, a system of $4 \times 4 \times 4$ unit cells of the structure $C 15$ is created, which is equivalent to 1024 magnetic atoms. The size of the system is large enough to discuss the first-order phase transition, where long-range spin-spin correlations are absent. An interphase boundary is introduced which divides the system in two equal parts, one magnetic and one non-magnetic. The magnetic part is in one of 90 ground state structures. During the simulation of the phase transition, magnetic state of non-magnetic atoms is determined subsequently, in order of their distance to the interphase boundary. The automaton applied is for $\Delta=0$, and for small positive or zero magnetic field. The structure which appears does depend on the initial structure, the angles between the normal to the interphase boundary and the system coordinates, and the value of magnetic field. We can compare this structure, and in particular its energy, with that of the initial ground state structure.

In the second part of the simulation we change the magnetic state of one magnetic atom at the interphase boundary. Then we compare the obtained magnetic structures for both orientations of this atom. The Hamming distance between these two configurations informs us on the stability of the structure formed with

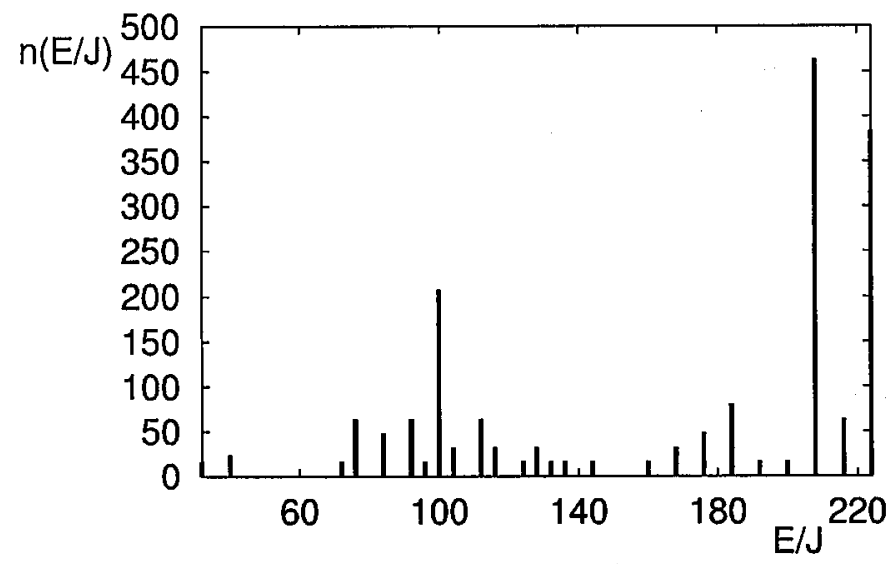

Fig. 1. Histogram of energies of metastable structures, obtained for zero field. 
respect to point damages. In both parts of the simulation we have applied open boundary conditions. Results are gathered from the internal 8 unit cells.

We find that the structure formed during the transition is always different from the initial structure. For positive magnetic field, the energy of the structure is equal to the ground state energy, i.e. $16 \mathrm{~J}$ per unit cell. For zero magnetic field, the energy of the formed structure is always positive. In Fig. 1 we show the histogram of energies obtained in the latter case, which is calculated from the simulations with 90 ground state configurations of the initial structure and with 20 directions of the interphase boundary.

The simulations of damage spreading for positive magnetic field confirms the fact that the energy of damaged structure is equal to the ground state energy. The only contribution is from the point damage, and it is equal to $\pm 2 J$. For zero magnetic field, non-magnetic atoms enhance the energy again. Although the size of a damage is about 20 sites in the average, its energy never excesses $14 J$, and the average of energy is less than $6 J$.

\section{Discussion}

The model approach presented here is not directly applicable to $\mathrm{YMn}_{2}$. Experimental data [4] show that the ground state degeneracy is at least partially removed by tetragonal distortion of 0.5 percent. Also, $\mathrm{Mn}$ magnetic moments are helically oriented, and the Blume-Capel model is only an approximation. Still the magnetic structure of $\mathrm{YMn}_{2}$ is not known [5]. Neutron measurements show thermal hysteresis [4], which indicates that the history of the sample can be relevant. The spectra can be interpreted as resulting from a superposition of two helical components [4], from a mixed phase where some of $\mathrm{Mn}$ atoms remain non-magnetic [6], or from a mixture of magnetic and non-magnetic phases [7]. It is well probable that the degeneracy of the ground state is relevant for this material.

Here, the ground state of antiferromagnetic $C 15$ structure is found to be highly degenerated. Our results show that translational symmetry of the magnetic structure cannot be expected. None of the periodic ground states is reproduced during the transition, and the energy of the interface between particular periodic structures is zero. Moreover, the structure which is the actual output of the transition is found to be unstable with respect to point damages. Although the simulations are performed for $\Delta=0$, an inclusion of this term can lead to the stabilization of magnetic moments.

\section{References}

[1] M. Shiga, Physica B 149, 293 (1988).

[2] K. Kułakowski, A. del Moral, Phys. Rev. B 56, 8928 (1997).

[3] K. Kułakowski, M. Antoniuk, J. Deskur, P. Gawroniski, J. Magn. Magn. Mater. 196-197, 824 (1999).

[4] R. Cywinski, S.H. Kilcoyne, C.A. Scott, J. Phys. Condens. Matter 3, 6473 (1991).

[5] J.M. de Teresa, C. Ritter, M.R. Ibarra, Z. Arnold, C. Marquina, A. del Moral, J. Phys. Condens. Matter 8, 8385 (1996).

[6] R. Ballou, C. Lacroix, M.D. Nunez Regueiro, Phys. Rev. Lett. 66, 1910 (1991).

[7] M.R. Ibarra, L. Garcia-Orza, A. del Moral, Solid State Commun. 84, 875 (1992). 